\title{
A Coherent Accumulation Detection Method Based on SA-DPT for Highly Maneuvering Target
}

\section{Cunsuo Pang ( $\nabla$ pangcunsuo@126.com )}

North University of China https://orcid.org/0000-0002-8523-5196

\section{Hualing Guo}

North University of China

\section{Huiling Hou}

North University of China

\section{Research}

Keywords: highly maneuvering target, weak signal detection, scaling algorithm(SA), discrete polynomial phase Transform(DPT)

Posted Date: October 11th, 2021

DOl: https://doi.org/10.21203/rs.3.rs-952438/v1

License: @ (i) This work is licensed under a Creative Commons Attribution 4.0 International License. Read Full License 


\title{
A Coherent Accumulation Detection Method Based on SA-DPT for Highly Maneuvering Target
}

\author{
Cunsuo Pang ${ }^{1}$, Hualing Guo ${ }^{1}$, Huiling Hou $^{1, *}$ \\ ${ }^{1}$ National Key Laboratory of Electronic Measurement Technology, North University of China, Taiyuan 030051, China
}

\begin{abstract}
The emergence of highly maneuverable weak targets has led to a serious degradation or even failure of traditional radar detection. In this paper, a coherent accumulation algorithm based on combination of scaling algorithm (SA) and discrete polynomial-phase Transform (DPT) is proposed in terms of the calculation burden and detection performance, which can, firstly, perform fewer times of speed parameter compensation based on SA for the transmitted signal; secondly, use segmented FFT to estimate the time delay range of the target initial distance for the echo signal, and then use the DPT algorithm to complete the parameter estimation such as target speed and acceleration within the estimated time delay unit, while analyzing the effects of the number of segments, compensation speed and delay unit on the detection performance, and giving out the output SNR and the amount of complex multiplication of the proposed algorithm. Finally, the proposed algorithm has been verified by experimental data for its effectiveness in accumulation gain and parameter estimation. This method is for sub-optimal estimation, requiring much less computation than full-parameter search methods, but performs better than non-parametric search methods in detecting weak signals.
\end{abstract}

Keywords highly maneuvering target,weak signal detection, scaling algorithm（SA）,discrete polynomial phase Transform (DPT)

\section{INTRODUCTION}

With the development of aircraft technology, more and more aircraft have the characteristics of high speed, high maneuverability and stealth, which brings great challenges to conventional radar detection. With the same observation time, the echo accumulation energy of a highly maneuverable weak target is lower than that of a conventional one. To improve the detection probability of targets, a commonly used method to increase the accumulation time is coherent accumulation[1-6]. There have been two main categories of long-time coherent accumulation methods so far. One is joint search methods based on parameter, and the other is cross-correlation parameter estimation methods based on order-reduction processing. Among them, [7] studied the parameter compensation methods for speed and acceleration based on SA,improving the probability of target detection. [8] studied the parameter estimation methods for distance, speed, and acceleration based on Maximum likelihood estimator (MLE), and analyzed the parameter errors using the Cramer-Raw lower bound theory. [9]-[12] studied the compensation method for range migration (RM) based on the keystone (KT) method, but there were velocity ambiguity numbers for the target with higher velocity. [13] [16] studied a highly maneuvering target detection methods based on Radon fourier transform (RFT), which used Radon transform and FT to complete RM compensation * Corresponding author: Huiling Hou

E-mail addresses:hou_huiling@126.com and Doppler frequency migration compensation, respectively. [17] studied the detection method for highly maneuvering targets based on Three-dimensional scaled transform (TDST), whose output signal-to-noise had improved by more than $10 \mathrm{~dB}$ compared with that of the detection method for moving targets. [18] studied the RM compensation based on Radon transform and Doppler frequency migration compensation based on Lv's distribution (LVD). The detection performance of this method was close to RFT. [19]-[22] studied the coherent accumulation method for high speed target based on FRFT. [23]-[24] studied the sparse Fourier transform (SFT) method to accelerate the speed compensation and spectrum computation of high-speed targets. [25]-[28] studied the highly maneuvering target detection methods based on the thoughts of Discrete polynomial-phase Transform (DPT). [29]-[33]studied the highly maneuvering target detection methods based on the thoughts of adjacent cross correlation function (ACCF).

Among the above methods, the first type of method(e.g. MLE,KT,FRFT) has high accumulation gain and good detection performance for low SNR signals, but has larger calculation burden and poorer real-time performance; the second type of method(e.g. DPT,ACCF) has the advantage of lower computational complexity than that of the first type of method, but requires a higher input SNR than that 
of the first type of method and is not suitable for detecting weak target signals.

Considering both the improvement of the detection performance for weak target signals and the reduction of calculation burden, this paper focuses on analyzing the reasons why the DPT method reduces the detection ability of weak target signals, finding out that, while decreasing the calculation burden, DPT algorithm increases the effect of noise to lower the output SNR. In order to further improve the detection performance of the DPT method for weak target signals, a coherent accumulation detection method based on combination of SA-DPT for highly maneuvering targets has been proposed in this paper, with its structure arranged as follows: The first part that overviews the long time coherent accumulation method commonly used for weak signal detection, and analyzes its detection performance and calculation burden; the second part that gives out the maneuvering target echo model based on the LFM radar; the third part that analyzes the factors affecting the detection performance of DPT algorithm, and proposes a long time coherent accumulation method based on the combination of speed compensation, segmented FFT, and DPT algorithm; the fourth part that analyzes the effects of segmented parameters, speed compensation parameters, and delay units on detection performance, and compares the complex multiplication burdens of different algorithms; the fifth part that verifies the effectiveness of the proposed method by experimental data and the sixth part that summarizes and discusses the full text.

\section{HIGH MANEVERING TARGRT ECHO MODEL}

Assuming that the detection system is an LFM pulse radar, the frequency spectrum of the transmitted and received signal after down-conversion can then be expressed as ${ }^{[19]}$ :

$$
\begin{aligned}
& R(f, n)=\frac{1}{\sqrt{\mu}} \operatorname{rect}\left(\frac{f}{\mu T_{0}}\right) \exp \left(-j \pi \frac{f^{2}}{\mu}\right) \\
& S(f, n)=\frac{A_{0}}{\sqrt{\mu}} \operatorname{rect}\left(\frac{f-f_{d}}{\mu T_{0}}\right) \exp \left[-j \pi \frac{\left(f-f_{d}\right)^{2}}{\mu}\right] \\
& \times \exp \left(j 2 \pi f_{d} n T\right) \exp \left(-j \frac{4 \pi R_{0}}{\lambda}\right) \\
& \times \exp \left[j \pi \alpha_{d}(n T)^{2}\right] \exp \left[-j 2 \pi\left(f-f_{d}\right)\left(\tau_{0}-\beta_{0} n T\right)\right]
\end{aligned}
$$

where, $A_{0}$ is the amplitude of the signal, $\mu$ is frequency modulation rate, $T_{0}$ is pulse width, $R_{0}$ is the initial range of the target distance from radar, $\tau_{0}=2 R_{0} / c$ is the initial time delay of the target, $\beta_{0}=2 v_{0} / c$ is the time delay change rate of the target, $f_{d}=2 v_{0} / \lambda$ is the Doppler frequency of the target, $\alpha_{d}=2 a / \lambda$ is the first-order term of the Doppler frequency change, $v_{0}$ is the target radial speed, $a$ is the target radial acceleration, $T$ is pulse repetition period, $c$ is velocity of light, $f_{c}$ is signal carrier, $\lambda=c / f_{c}$ is the transmitted signal wavelength.

Multiplying Equation(1) by (2) to arrive at the frequency domain and time domain signals after pulse compression of the echo signal as:

$$
\begin{aligned}
& X(f, n) \approx \frac{A_{0}}{\sqrt{\mu}} \operatorname{rect}\left(\frac{f}{B}\right) \exp \left[-j 2 \pi f\left(\tau_{0}-\beta_{0} n T\right)\right] \exp \left(-j \frac{4 \pi R_{0}}{\lambda}\right) \\
& \cdot \exp \left[j 2 \pi f_{d}\left(n T+\tau_{0}-\beta_{0} n T\right)\right] \exp \left[j \pi \alpha_{d}(n T)^{2}\right] \\
& \left.x(t, n) \approx A_{0} \sqrt{D} \sin c\left[\pi B(t)-\tau_{0}+\beta_{0} n T+\frac{f_{d}}{\mu}\right)\right] \exp \left(-j \pi \frac{f_{d}^{2}}{\mu}\right) \\
& \cdot \exp \left(j \pi f_{d}\left(t+\tau_{0}-\beta_{0} n T+\frac{f_{d}}{\mu}\right)\right) \exp \left(j 2 \pi f_{d} n T\right) \exp \left(j \pi \alpha_{d}(n T)^{2}\right)
\end{aligned}
$$

where, $D=B T_{0}$ is the product of the time width and the bandwidth, $B$ is the bandwidth of input signals.

It can be seen from Equation(3) and (4) that the exponential functions that cause the target envelope to produce delay walk and Doppler frequency walk are:

$$
\begin{aligned}
& R(\widehat{f}, v)=\exp \left[-j 2 \pi \widehat{f} \tau_{0}-\Delta \tau\right] \\
& F\left(f_{d}, a\right)=\exp \left[j 2 \pi f_{d}(1+\Delta f)\right]
\end{aligned}
$$

where,

$$
\begin{aligned}
& \Delta \tau \approx \beta_{0} n T \\
& \Delta f \approx \alpha_{\mathrm{d}}(n T)
\end{aligned}
$$

Equation (7) to (8) show that in the same accumulation time, the greater the target speed and acceleration, the more serious the target energy diffusion, and therefore the detection probability will fall significantly. The time-delay migration caused by velocity and the Doppler frequency migration caused by acceleration have been mainly considered in this paper.

\section{COHERENT ACCUMULATION METHOD BASED ON SA-DPT}

3.1 ANALYSIS OF FACTORS AFFECTING THE DETECTION PERFORMANCE OF DPT

When cross-correlation processing is performed on Equation (3) by column, it can obtain ${ }^{\text {[28-31]: }}$

$$
\begin{aligned}
& C\left(\widehat{f}, n, \tau_{N}\right)=X(\widehat{f}, n) * X\left(\widehat{f}, n-\tau_{N}\right) \\
& =A_{1} \operatorname{rect}\left(\frac{\widehat{f}}{B}\right) \exp \left[j 2 \pi \widehat{f}\left(\beta_{0} \tau_{N} T-2 \tau_{0}\right)\right] \exp \left[j 2 \pi \alpha_{d} \tau_{N} T(n T)\right]+w\left(\widehat{f}, n^{\prime}\right)
\end{aligned}
$$

where, $A_{1}=\frac{A_{0}^{2}}{\mu} \exp \left(-j \frac{8 \pi R_{0}}{\lambda}\right) \exp \left(j 4 \pi f_{d} \tau_{0}\right) \exp \left[-j \pi \alpha_{d}\left(\tau_{N} T\right)^{2}\right], w$ represents a mean value of 0 , the variance is the white Gaussian noise of $\sigma^{2}, \tau_{N}$ is the delay unit relative to $\mathrm{N}, n^{\prime} \in\left[1, N-\tau_{N}\right]$.

When performing IFFT and FFT processing on $f, n$ in (9) respectively, it can obtain:

$$
\begin{aligned}
& C\left(\widehat{t}, f_{a}, \tau_{N}\right)=\mathrm{FFT}_{n}\left[\operatorname{IFFT}_{\widehat{f}} C\left(\widehat{f}, n, \tau_{N}\right)\right] \\
& =\mathrm{A}_{1}\left(N-\tau_{N}\right) \operatorname{sinc}\left[B\left(\widehat{t}-\beta_{0} \tau_{N} T\right)\right] \sin c\left[T_{\tau_{N}}\left(f_{a}-\alpha_{d} \tau_{N} T\right)\right]+w\left(\widehat{t}, n^{\prime}\right)
\end{aligned}
$$


(10)

where, $T_{\tau_{N}}=\left(N-\tau_{N}\right) T$.

According to Equations (3)-(10), the echo output power, noise power, and output SNR can be obtained and expressed respectively as ${ }^{[26]}$ :

$$
\begin{aligned}
\left.P_{\text {out }}\right|_{\text {signal }}=\left(N-\tau_{N}\right)^{2} A_{0}^{4} \\
\left.P_{\text {out }}\right|_{\text {noise }}=\sum_{n=1}^{N-\tau_{N}}\left|A_{0}^{2} \delta^{2}+\delta^{2}+\delta^{4}\right|=\left(N-\tau_{N}\right)\left(2 A_{0}^{2} \delta^{2}+\delta^{4}\right) \\
S N R_{\text {out }}=\frac{\left.P_{\text {out }}\right|_{\text {signal }}}{\left.P_{\text {out }}\right|_{\text {noise }}} \\
=\left.\frac{M N}{2} \frac{S N R_{\text {in }}^{2}}{1+2 S N R_{\text {in }}}\right|_{\tau_{N}=\frac{1}{2}}, S N R_{\text {in }}=A_{0}^{2} / \delta^{2}
\end{aligned}
$$

where, $\left.P_{\text {out }}\right|_{\text {signal }},\left.P_{\text {out }}\right|_{\text {noise }}$ are the power of the output signal and the noise power respectively.

When comparing Equation (13) with the output SNR of the MLE method in [8], it can yield:

$$
\begin{aligned}
S N R_{\text {ratio }}= & \frac{\left.\frac{M N}{2} \frac{S N R^{2}{ }_{i n}}{1+2 S N R_{\text {in }}}\right|_{\tau_{N}=\frac{1}{2}}}{M N S N R_{\text {in }}} \\
= & \left.\frac{1}{2} \frac{S N R_{\text {in }}}{1+2 S N R_{\text {in }}}\right|_{\tau_{N}=\frac{1}{2}}
\end{aligned}
$$

From (14), when $\mathrm{SNR}_{\text {in }}$ is smaller, it can yield:

$$
\left.S N R_{\text {ratio }} \approx \frac{1}{2} S N R_{i n}\right|_{\tau_{N}=\frac{1}{2}}
$$

It can be seen from (15) that when $\mathrm{SNR}_{\text {in }}$ is smaller, the detection performance of the DPT method is approximately $10 \log _{10}\left(\mathrm{SNR}_{\text {in }} / 2\right)$ lower than that of the MLE method. Figure 1 shows the loss of SNR caused by (15) at different low SNRs. As can be seen from Figure 1, when the input SNR is lower than 0.2, the output SNR loss of (15) will be greater than $10 \mathrm{~dB}$. Therefore, the detection performance of DPT for weak signals is poor.

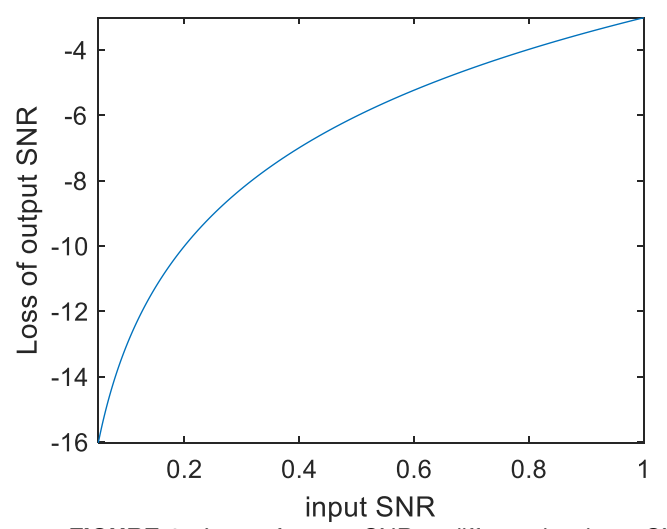

It can be seen from (12) that after DPT processing, the noise power increases and the output SNR decreases. The analysis finds out that the noise power is related to the length of signal processing, where the noise processing range can be reduced, $S N R_{\text {out }}$ can be increased. To this end, a coherent accumulation method based on the combination of SA speed compensation and segmented FFT is presented in this paper to obtain the initial position of the target, and then use the DPT algorithm to complete the estimation of the target parameters.

\subsection{THE SPEED COMPENSATION METHOD BASED ON SA FOR TRANSMITTED SIGNAL}

In order to obtain the initial distance range of the target, the echo envelope RM caused by $\sin c\left[\pi B\left(t-\tau_{0}+\beta_{0} n T+\frac{f_{d}}{\mu}\right)\right]$ in Equation (4) can be first calibrated, and the efficiency of the algorithm can be considered to be improved at the same time. In this paper, the SA method is used to compensate the speed of the transmitted signal.

Equation (1) (7) suggest that the factor that causes the target echo envelope to walk is the exponential function term $\exp (-j 2 \pi \hat{f} \Delta \tau)$, Therefore, after speed compensation on Equation (1), its expression can be written as:

$$
R^{\prime}\left(f^{\prime}, n\right)=R(\hat{f}, n) * \omega\left(f^{\prime}, n\right)
$$

where,

$$
\begin{aligned}
\omega\left(f^{\prime}, n\right) & =\exp \left(j 2 \pi f^{\prime} \Delta \tau\right) \\
& =\exp \left(-j 2 \pi f^{\prime} \beta_{0}^{\prime} n T\right)
\end{aligned}
$$

In (17), $\beta^{\prime}=\frac{29 \%}{c}, 1 \%$ is the velocity compensation value.

Substituting (16) for (1), Equation (4) can be rewritten as:

$$
\begin{aligned}
& x(t, n) \approx A_{0} \sqrt{D} \sin c\left[\pi B\left(t-\tau_{0}+\Delta \beta n T+\frac{f_{d}}{\mu}\right)\right] \exp \left(-j \pi \frac{f_{d}^{2}}{\mu}\right) \\
& \cdot \exp \left(j \pi f_{d}\left(t+\tau_{0}-\Delta \beta n T+\frac{f_{d}}{\mu}\right)\right) \exp \left(j 2 \pi f_{d} n T\right) \exp \left(j \pi \alpha_{d}(n T)^{2}\right)
\end{aligned}
$$

where, $\Delta \beta=\beta_{0}-\beta_{0}^{\prime}$.

In (18), the closer when $\beta_{0}$ is approching to $\beta_{0}^{\prime}$, the smaller the RM of the echo envelope is, which is more conducive to the estimation of the initial position of the target. However, when the echo input SNR is lower, in order to further improve the estimation of the target position, a method based on segmented FFT is employed in this paper to improve the output SNR of the signal.

\subsection{POSITION ESTIMATION METHOD BASED ON SEGMENTED FFT}

Suppose that $\mathrm{N}$ echo signals are divided into $\mathrm{L}$ segments, and $\mathrm{P}$ is the number of points in each segment. After performing segmented FFT on (18) by column, the frequency spectrum corresponding to the signal amplitude in the $l$-th segment can be expressed as:

FIGURE 1. Loss of output SNR at different low input SNR 


$$
\begin{aligned}
& z\left(t, f_{l}\right) \approx A_{0} \sqrt{D} \mid \sum_{p=1}^{P} \sin c\left[\pi B\left(t-\tau_{0}+\Delta \beta p^{\prime} T+\frac{f_{d}}{\mu}\right)\right] \\
& \cdot \exp \left[j 2 \pi\left(f_{d}-f_{l}\right) p^{\prime} T\right] \exp \left[j \pi \alpha_{d}\left(p^{\prime} T\right)^{2}\right] \mid, l=1,2, \Lambda L
\end{aligned}
$$

where, $p^{\prime}=p+(l-1) \mathrm{P}, p \in[1, \mathrm{P}]$ is the mark for the segment index.

From (19), the phase of the p-th point in the $l$-th signal segment can be expressed as:

$$
\varphi_{p, l}=2 \pi\left\{f_{d}[p+(l-1) P] T+0.5 \alpha_{d}[p+(l-1) P]^{2} T^{2}\right\}
$$

From (20), the frequency of the p-th point in the $l$-th signal segment is:

$$
\begin{aligned}
f_{p, l} & =\frac{1}{2 \pi} \frac{\Delta \varphi_{p, l}}{\Delta(p T)} \\
& =f_{d}+\alpha_{d}[p T+(l-1) P T]
\end{aligned}
$$

From (21), it can be known that the signal frequency relationship of the adjacent $\Delta \mathrm{p}$ points in the $l$-th segment of the signal is:

$$
\begin{aligned}
\Delta f_{\Delta p, l} & =\Delta f_{p+\Delta p, l}-\Delta f_{p, l} \\
& =\alpha_{d} \Delta p T \\
& \leq \alpha_{d} P T, p \in[1, P]
\end{aligned}
$$

According to Equation (20)-(22) and $\alpha_{d} P T \leq \frac{1}{P T}$, can be expressed as:

$$
z\left(t, f_{l}\right) \approx A_{0} \mathrm{P} \sqrt{D} \sin c\left[\pi B\left(t-\tau_{0}+\Delta \beta p^{\prime} T+\frac{f_{d}}{\mu}\right)\right] \sin c\left[\mathrm{PT}\left(f_{d}-f_{l}\right)\right]
$$

Comparing Equation (4) and (23), it can be seen that if the time delay walk of $\sin c\left[\pi B\left(t-\tau_{0}+\Delta \beta p^{\prime} T+\frac{f_{d}}{\mu}\right)\right]$ can be eliminated, the amplitude of the echo pulse compression can be increased by $\mathrm{P}$ times at the maximum, which can further improve the estimation of the initial position of the target.

After optimizing the maximum value of (23), the initial time delay position of the target can be estimated based on the parameters corresponding to the peak value. This process is expressed as:

$$
\Delta \tau=\arg \max _{l}\left[z\left(t, f_{l}\right)\right]
$$

where, $\Delta \tau$ is expressed as the time delay estimation of the initial position of the target.

The estimated time delay range of the target based on (24) is:

$$
\Delta \tau^{\prime} \in[\Delta \tau-b, \Delta \tau+b]
$$

where, $b$ can be set according to Equation (7).

\subsection{TARGET PARAMETER ESTIMATION BASED ON DPT}

Considering the influence of noise, the target delay range obtained by (24) is substituted into (10), and the following is obtained:

$$
\begin{aligned}
& C^{\prime}\left(\dot{t}, f_{a}, \tau_{N}\right)=\mathrm{A}_{1}\left(N-\tau_{N}\right) \sin c\left[\pi B\left(t-\Delta \beta^{\prime} \tau_{N} T\right)\right] \\
& \cdot \sin c\left[T_{\tau_{N}}\left(f_{a}-\alpha_{d}^{\prime} \tau_{N} T\right)\right]+\omega\left(\Delta \tau^{\prime}, N-\tau_{N}\right)
\end{aligned}
$$

where, $w\left(\Delta \tau, n^{\prime}\right)$ represents the time delay processing range of the target, and $\Delta \beta^{\prime}=\frac{2 v^{\prime}}{c}, \Delta \alpha_{d}^{\prime}=\frac{2 a^{\prime}}{c}, \mathcal{v}^{\prime}$ and $a^{\prime}$ are the estimated values of target speed and acceleration respectively.

According to the results of (18) and (26), the values of the target velocity and acceleration can be approximately expressed respectively as: ${ }^{[28]}$ :

$$
\begin{aligned}
& \hat{v}^{\prime}=\frac{c}{2 \tau_{N} T} \frac{1}{f_{s}} \Delta m+\mathscr{Q} \\
& a^{\prime}=\frac{\lambda}{2 T^{2}} \frac{1}{\left(N-\tau_{N}\right) \tau_{N}} \Delta n
\end{aligned}
$$

where, $\Delta m, \Delta n$ respectively represent the time delay position and Doppler frequency position, and $\%$ is the compensation value of the speed in (18).

According to $\dot{f}^{\prime}$ and $\dot{a}^{\prime}$, the compensation function $\exp \left(-j 2 \pi f \frac{2 \hat{v}^{\prime}}{c} n T\right) \exp \left[-j \pi \frac{2 \widehat{a}^{\prime}}{c}(n T)^{2}\right]$ is constructed, and is substituted into the Equation (3), the parameters such as the distance and speed of the target can be obtained by using the MTD method.

The implementation process of the above algorithm is shown in Figure 2.

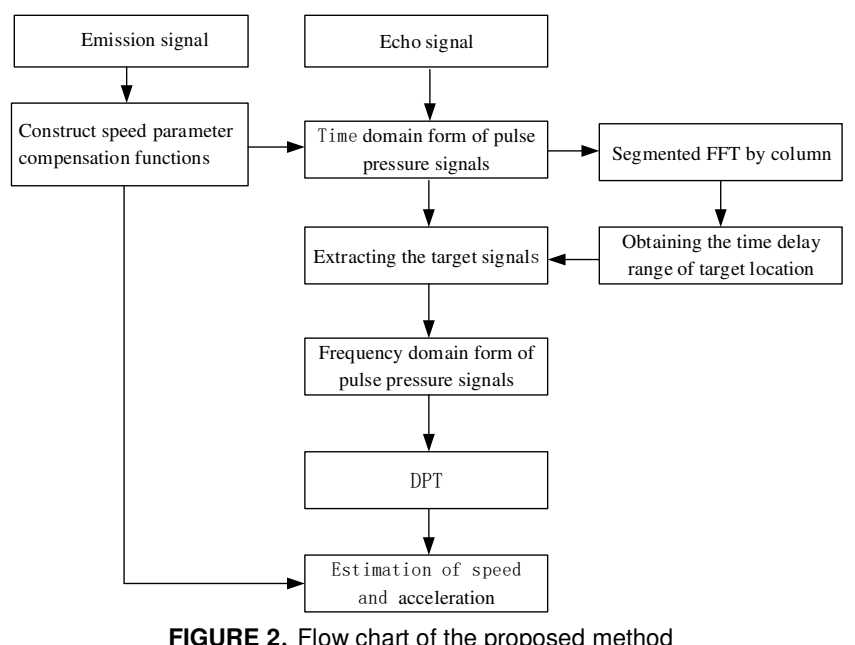

\section{ANALYSIS ON ALGORITHM PERFORMANCE}

\subsection{Analysis ON OUTPUT SNR}

It can be obtained from Equation (11)-(12) that the signal output $\left.P_{\text {out }}\right|_{\text {signal }}$ in (26) and (10) has not changed, but the noise output $\left.P_{\text {out }}\right|_{\text {noise }}$ has changed, namely:

$$
\left.P_{\text {out }}^{\prime}\right|_{\text {noise }}=\sum_{n=1}^{N-\tau_{N}}\left|A_{0}^{2} \delta^{\prime 2}+\delta^{\prime 2}+\delta^{\prime 4}\right|=\left(N-\tau_{N}\right)\left(2 A_{0}^{2} \delta^{\prime 2}+\delta^{\prime 4}\right)
$$

where, $\delta^{\prime 2}$ in (29) is affected by (25), which can be expressed as: 


$$
\delta^{\prime 2}=\frac{L_{\Delta \tau^{\prime}}}{M} \delta^{2}, L_{\Delta \tau^{\prime}}=2 b+1
$$

When the results of Equation (11), Equation (29) and Equation (30) are synthesized, resulting in that the output SNR of the signal in (26) can be expressed as:

$$
\begin{aligned}
S N R_{\text {out }} & =\frac{\left.P_{\text {out }}\right|_{\text {signal }}}{\left.P_{\text {out }}^{\prime}\right|_{\text {noise }}} \\
& =\left.\frac{N L_{\Delta \tau^{\prime}}}{2} \frac{S N R^{\prime 2}{ }_{\text {in }}}{1+2 S N R_{\text {in }}^{\prime}}\right|_{\tau_{N}=\frac{1}{2}}, S N R_{\text {in }}^{\prime}=A_{0}^{2} / \delta^{\prime 2}
\end{aligned}
$$

Considering that when $S N R^{\prime}{ }_{i n}$ is smaller, and comparing Equation (13) and (31), the output SNR can be improved as:

$$
\begin{aligned}
\Delta S N R & =\frac{\left.\Delta \tau^{\prime} S N R^{\prime 2}{ }_{i n}\right|_{\tau_{N}=\frac{1}{2}}}{\left.M S N R^{2}{ }_{i n}\right|_{\tau_{N}=\frac{1}{2}}} \\
& =\frac{M}{\Delta \tau^{\prime}}
\end{aligned}
$$

According to (32), it can be known that when $L_{\Delta \tau^{\prime}}$ is getting smaller, it can not only improve the output SNR, but also reduce the calculation burden at the same time. However, the improvement of the maximum effective SNR is limited by the accuracy of the maximum value of (24), when the maximum value detection deviates greatly from the true value, the effect of improving the SNR in (32) is reduced or even invalid.

Example 1: Let radar carrier frequency is $3 \mathrm{GHz}$, pulse repetition period is $3 \mathrm{~ms}$, pulse number is 128, signal bandwidth is $5 \mathrm{MHz}$, baseband sampling rate is $10 \mathrm{MHz}$, and the target, a point target, has a radial speed of $1000 \mathrm{~m} / \mathrm{s}$ and a radial acceleration of $10 \mathrm{~m} / \mathrm{s}^{2}$. Figure 3 shows the comparison of detection performance under different $L_{\Delta \tau^{\prime}}$, from which it can be seen that the smaller $L_{\Delta \tau^{\prime}}$ is, the better the detection performance. When the detection probability is $80 \%$, the requirement on the input SNR when $L_{\Delta \tau^{\prime}}$ is 20 is lower by about $5 \mathrm{~dB}$ when $L_{\Delta \tau^{\prime}}$ is 100 , which is more conducive to weak signal detection.

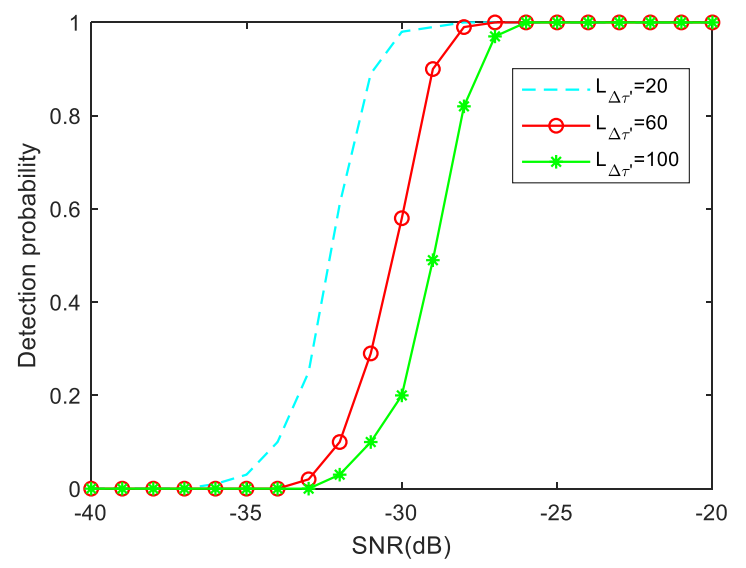

FIGURE 3. Comparison of detection performance under different $L_{\Delta \tau^{\prime}}$

\subsection{SELECTION OF $\tilde{v}$ AND P}

(1) Selection of speed compensation factor $\tilde{v}$

If the envelope walk is less than a distance unit, the relationship among the velocity compensation factor and the target velocity and accumulation time PT in (19) can be expressed as follows:

$$
\frac{\Delta v}{2} \mathrm{PT} \leq \frac{c}{2 f_{s}}, \Delta v=\vartheta \sigma v_{0}
$$

It can obtain from (33)

$$
\Delta v \leq \frac{c}{P T f_{s}}
$$

Let the target maximum speed is $\nu_{\max }$. The range of the speed compensation factor is then:

$$
\tilde{v} \in[0, \pm \Delta v, \Lambda, \pm Q \Delta v], Q=\left\lfloor\frac{v_{\max }}{\Delta v}\right\rfloor
$$

Equation (35) gives out the compensation speed range and step interval. For the sake of fast acquiring the compensation range of the speed value, the speed is searched using the binary search technique; then the $\mathrm{Q}$ in (35) can be rewritten as:

$$
Q^{\prime}=\left\lfloor\frac{\nu_{\max } / 2^{z}}{\Delta \nu} \mid * \Delta \nu, z=1,2,3, \cdots \cdots\right.
$$

(2) Selection of $P$

In order to ensure the establishment of (23), the relationship among the signal length $\mathrm{P}$, target acceleration $\mathrm{a}$ and sampling interval $\mathrm{T}$ in (19) should meet the following conditions:

$$
P \leq \frac{1}{T \sqrt{\alpha_{d}}}=\frac{1}{T} \sqrt{\frac{c}{2 a f_{c}}}
$$

From (34) and (37) it can be seen that the length of $P$ is restricted by the speed and the acceleration , that is,

$$
P=\min \left[\frac{1}{T} \sqrt{\frac{c}{2 a f_{c}}}, \frac{c}{\Delta \nu T f_{s}}\right]
$$

Example 2: Both the radar and the target are supposed to have the same parameters as Example 1,the time delay range is $L_{\Delta \tau^{\prime}}$.Figure 4 shows a comparative analysis on the

detection performance of the proposed method with different $\tilde{v}$ and P. It can be seen from Figure 4(a) that with the same $P$, the closer when the speed compensation value is approaching to the true value, the better the detection performance; with the same speed compensation value, the detection performance is better when $\mathrm{P}=16$, because the selection of $\mathrm{P}$ is closer to the calculation result of (39). In addition, it can be seen that the detection performance is not much different when the speed compensation value is at $500 \mathrm{~m} / \mathrm{s}$ and $1000 \mathrm{~m} / \mathrm{s}$, which is beneficial to reduce the algorithm's search for the speed compensation value and improve the algorithm execution efficiency. Figure 4(b) shows that when the detection probability is $80 \%$, with the proposed method the input SNR is about $10 \mathrm{~dB}$ higher than with the MLE and about $5 \mathrm{~dB}$ lower than with the DPT, suggesting that the proposed method balances the detection 
performance and execution efficiency, thus conducive to the detection of weak target signals and the real-time realization of the method at the same time.

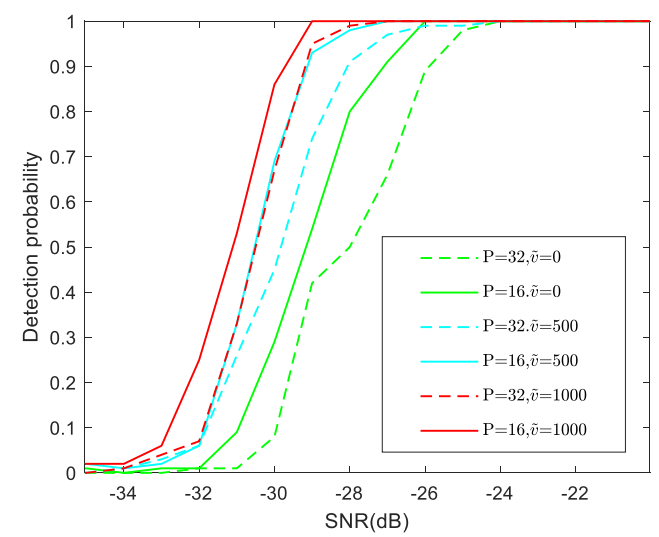

(a)

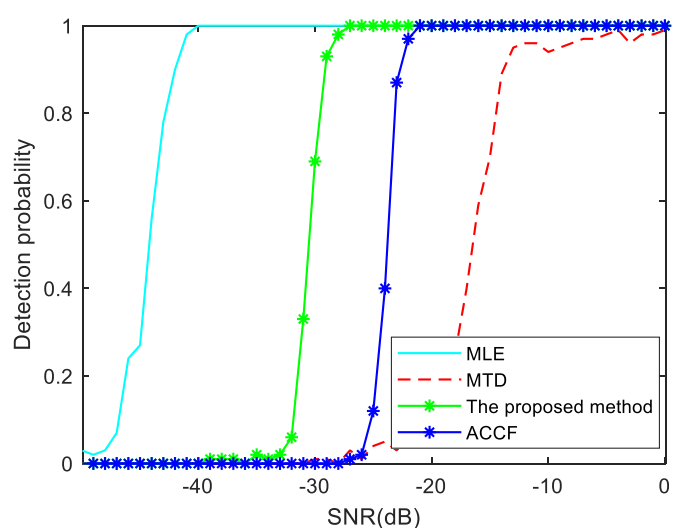

(b)

FIGURE 4. Comparative analysis on the detection performance of the proposed algorithm.(a)The detection performance of the proposed algorithm with differen $\tilde{\nu}$ and $\mathrm{P}$,(b)Comparison of detection performance of different algorithms.

\section{$\begin{array}{llllll}\text { 4.3 THE IMPACT OF } & \tau_{N} \text { ON DETECTION }\end{array}$}

\section{PERFORMANCE}

Let the simulation parameters be the same as example $1, L_{\Delta \tau^{\prime}}=20, \mathrm{P}=16$. Figure 5 shows the detection performance analysis of the proposed method with different $\tau_{N}$, from which it can be seen that the larger $\tau_{N}$ is, the greater the required input SNR with the same detection probability, but the smaller the relative estimation error of speed and acceleration is. On the contrary, the smaller $\tau_{N}$ is, the smaller the required input SNR is with the same detection probability, but the larger the relative estimation error of velocity and acceleration is. In order to facilitate the detection of weak signals, $\tau_{N}$ can be set as $0.3 \sim 0.5$.

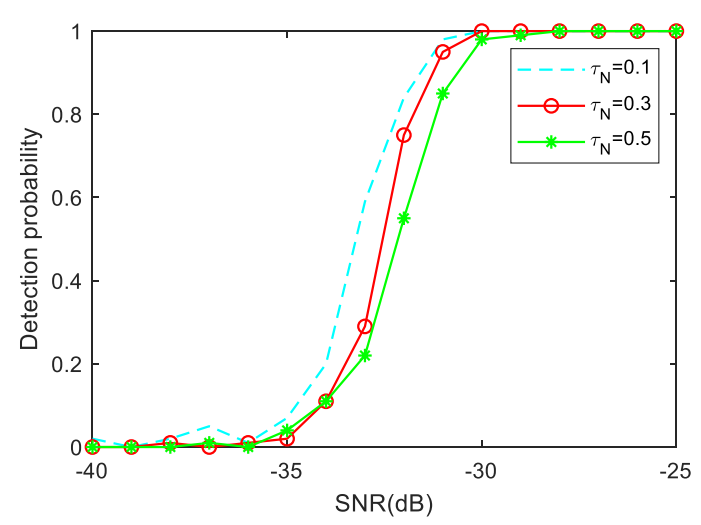

(a)

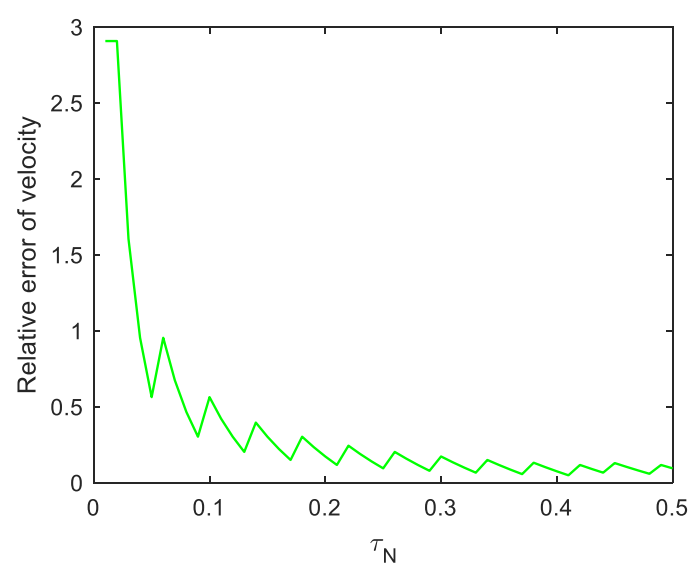

(b)

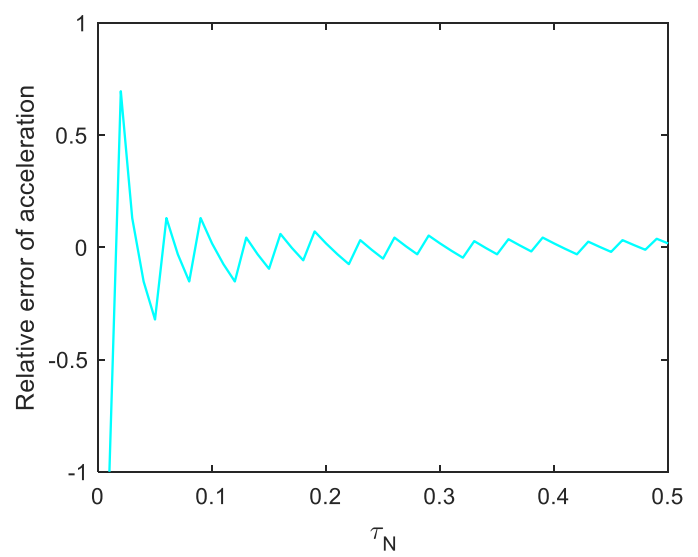

(c)

FIGURE 5. With different $\tau_{N}$, the detection performance and parameter estimation of the proposed algorithm.(a)Detection performance with different $\tau_{N}$, (b)Error of speed estimation,(c)Error of acceleration estimation

\subsection{COMPUTATION LOAD}

In what follows, the computational complexities of MLE, KT,TDST, ACCF, and the proposed method are analyzed . Assume that $\mathrm{M}$ and $\mathrm{N}$ denote the number of range cells and the number of pulses, $\mathrm{k}$ represents the number of speed compensation in MLE, KT, and $\mathrm{k}^{\prime}$ represents the number of speed compensation in the proposed algorithm, where $k^{\prime}<<k$.With the proposed algorithm, the phase 
compensation of the transmitted signal is before the pulse pressure, and therefore its calculated value can be stored in the register beforehand. The pulse pressure processing after one speed compensation requires $O\left(M N \log _{2} M\right)$ complex multiplication operation, and one cross-correlation operation involves $O\left(M N \log _{2} M N\right)$ complex multiplication operation, so the amount of complex multiplication required for $k^{\prime}$ times of speed compensation is approximately $O\left(k^{\prime} M N \log _{2} M N\right)$. The computational costs of the above-mentioned methods are listed in Table 1. Table 1 shows that, in addition to the ACCF, the proposed method has obvious advantages over other methods in terms of complex multiplication burden.

TABLE 1 Complex multiplication amount of different methods

\begin{tabular}{cl}
\hline Methods & Computational complexity \\
\hline MLE & $O\left(k N M^{2} \log _{2} N\right)$ \\
KT & $O\left(N M^{2}\right)+O\left(k^{2} N M \log _{2} M\right)$ \\
TDST & $O\left(N M^{2} \log _{2} N M\right)$ \\
ACCF & $O\left(N M \log _{2} M N\right)$ \\
Proposed method & $O\left(k^{\prime} N M \log _{2} M N\right)$ \\
\hline
\end{tabular}

\section{EXPERIMENTAL VERIFICATION AND ANALYSIS 5.1 SIMULATION EXPERIMENT}

(1)Single target detection performance

Suppose the radar system parameters be the same as Example 1,the target speed is $1000 \mathrm{~m} / \mathrm{s}$, the acceleration is $15 \mathrm{~m} / \mathrm{s}^{2}$, its distance from the radar is 100 kilometers, the SNR of the input signal is $-25 \mathrm{~dB}$, the speed compensation value is $500 \mathrm{~m} / \mathrm{s}$, and $\mathrm{p}=16$. Figure $5(\mathrm{a})$ gives the MTD processing result of the echo signal, Figure 5(b) gives the processing result by the DPT method, and Figure 5(c) shows the processing result by the method proposed. A comparison with the results in Figure 5 shows that the MTD method has caused obvious energy diffusion, both the ACCF and the proposed method can achieve the target detection, but the output SNR of the proposed method in this paper is larger.

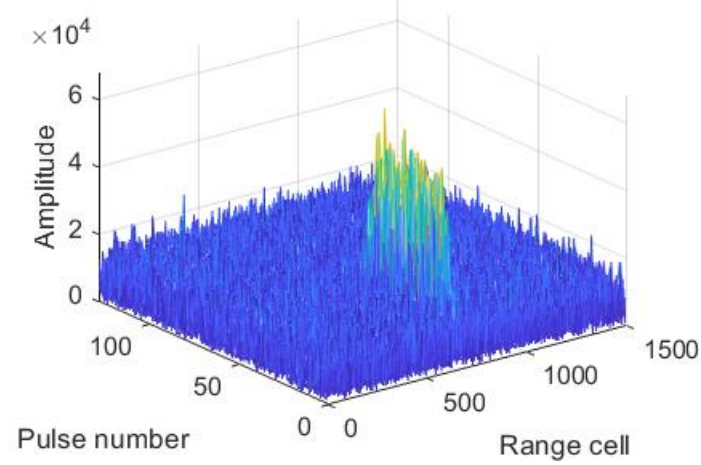

(a)

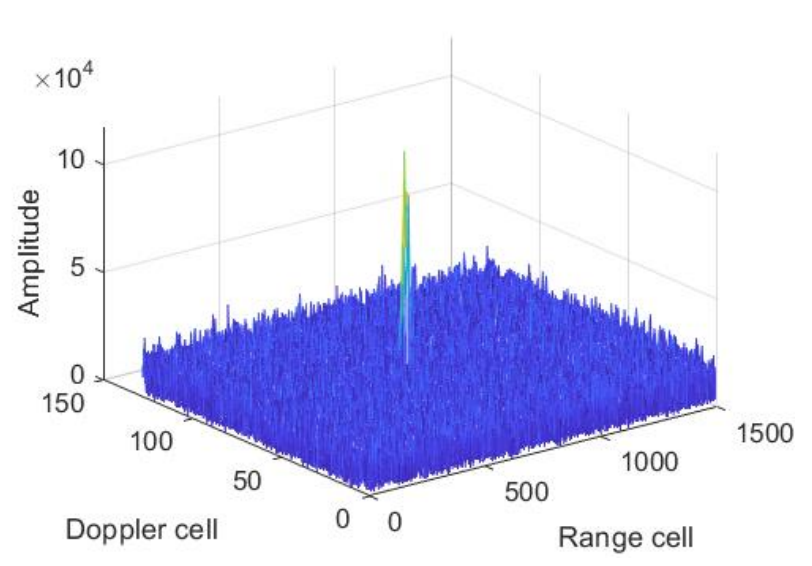

(b)

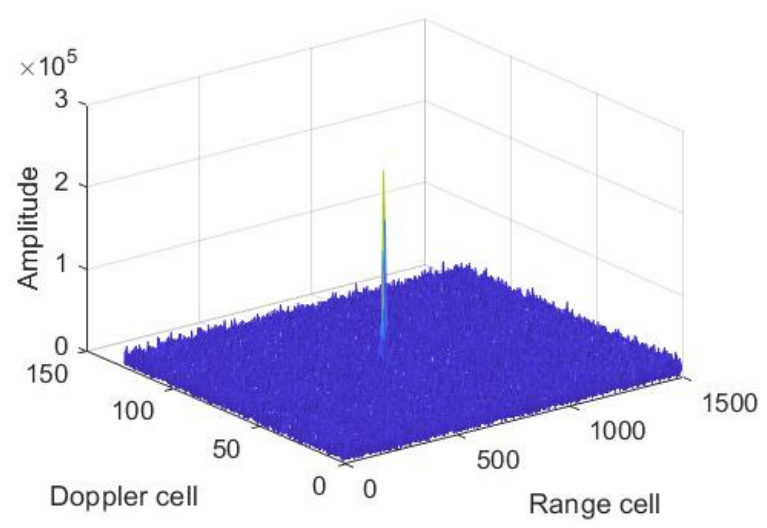

(c)

FIGURE 5. Single target detection performance by different algorithms.

(a) MTD , (b) ACCT, (c) Proposed method

(2)Multi-target detection performance

Two targets are assumed, with a speed of $1000 \mathrm{~m} / \mathrm{s}$, $1050 \mathrm{~m} / \mathrm{s}$, an acceleration of $5 \mathrm{~m} / \mathrm{s}^{2}, 10 \mathrm{~m} / \mathrm{s}^{2}$ respectively. The $\mathrm{SNR}$ of the input signals is $-25 \mathrm{~dB}$, and the speed compensation value is $500 \mathrm{~m} / \mathrm{s}, \mathrm{P}=16, L_{\Delta \tau^{\prime}}=100$. The other parameters are the same as in Example 1. Figure 6 shows

that MTD has caused serious energy diffusion. The ACCF method can detect two targets, but it suffers from Doppler spectrum spread. In contrast, the proposed method is capable of satisfactory detection of two targets and the target resolution is the highest among the several algorithms. 


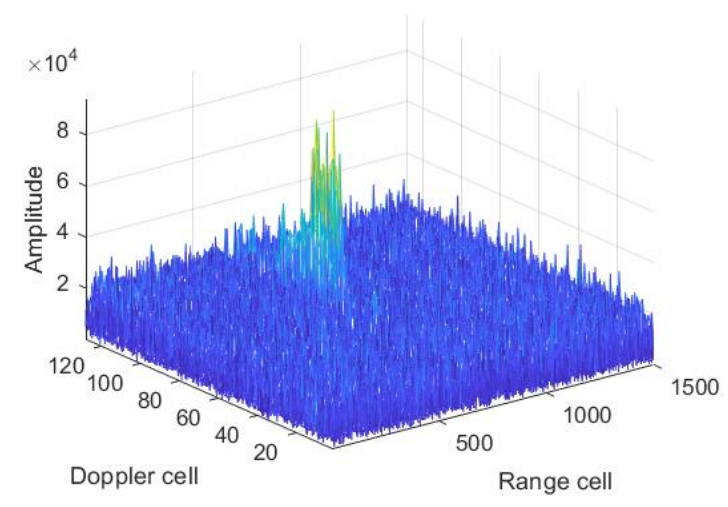

(a)

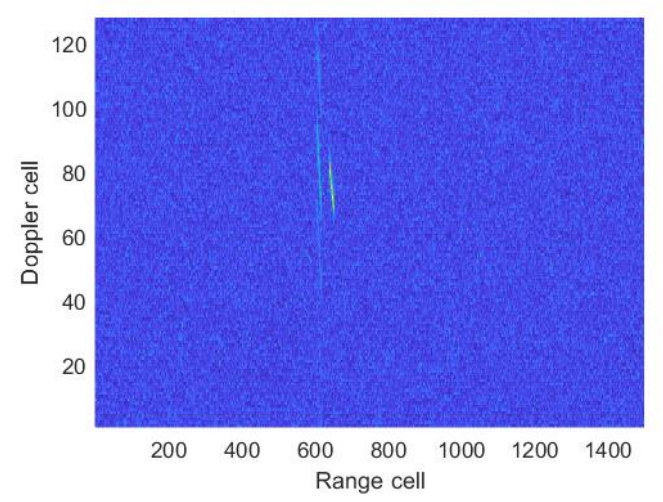

(b)

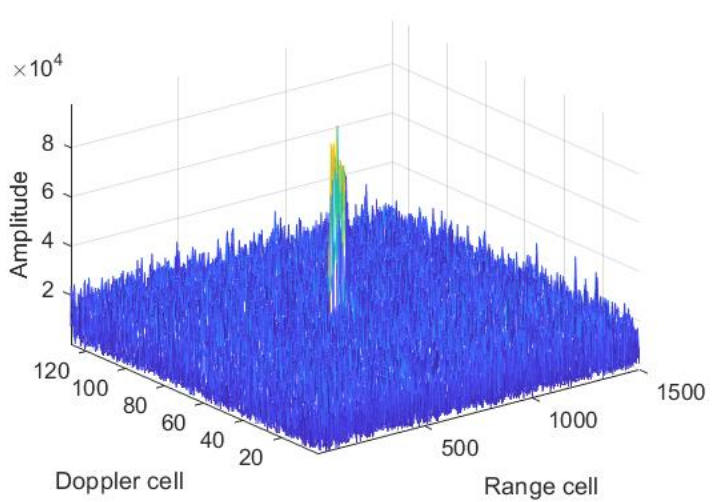

(c)

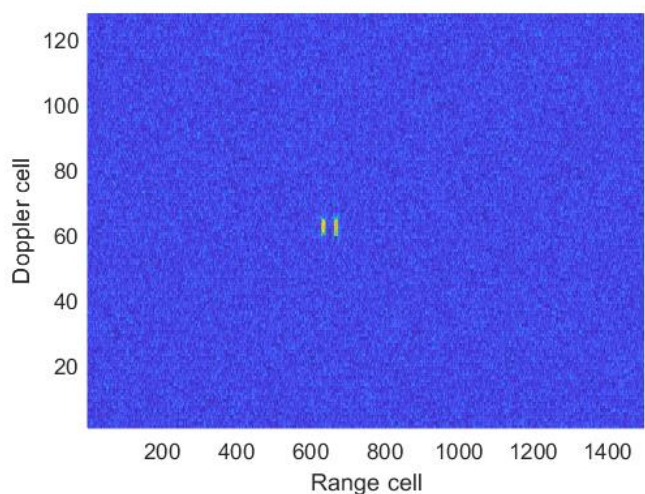

(d)

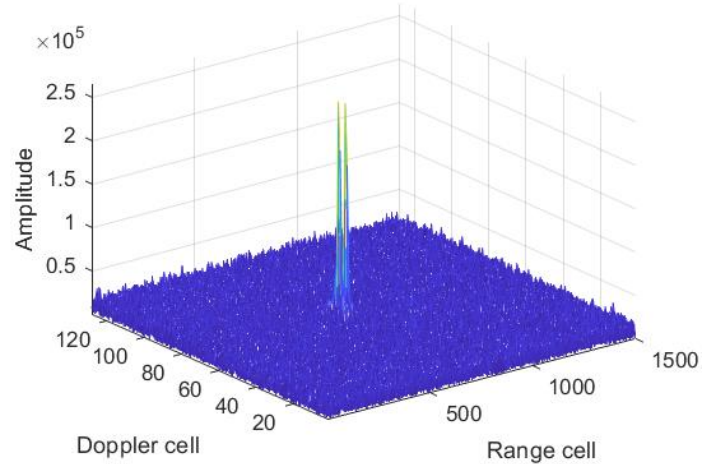

(e)

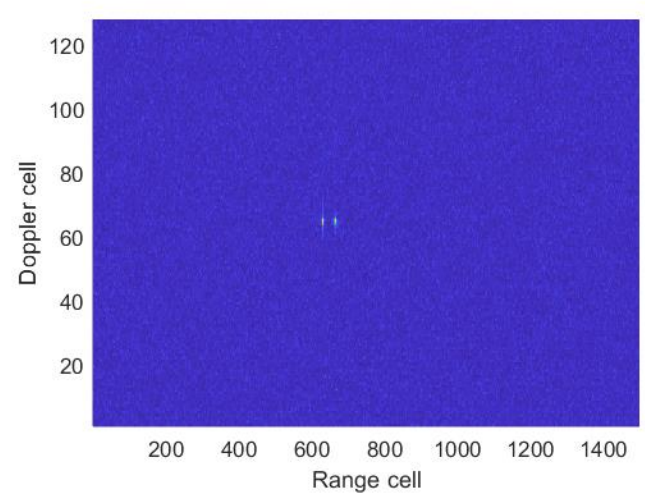

(f)

FIGURE 6. Dual target detection performance by different algorithms.

(a) MTD ,(b) Distance-Doppler dimension in Figure (a), (c) ACCF,

(d) Distance-Doppler dimension in Figure (c),(e) Proposed method, (f)

Distance-Doppler dimension in Figure (c)

The above simulation results show that the proposed algorithm can effectively detect weak signals, and its detection performance and operation efficiency are between the first type of coherent accumulation algorithm and the second type of coherent accumulation algorithm.

\subsection{VERIFICATION OF MEASURED DATA}

In order to further verify the effectiveness of the algorithm, the following measured data from aircraft were used for analysis, and the White Gaussian Noise that was added was 
$-25 \mathrm{~dB}$. The radar operated at the $\mathrm{S}$ band, the carrier frequency is $3 \mathrm{GHz}$, the signal bandwidth is $2 \mathrm{MHz}$, the pulse repetition time is $600 \mu \mathrm{s}$, the pulse duration is $60 \mu \mathrm{s}$, the integration pulse number is 2048, and the sampling frequency is $4 \mathrm{MHz}$, the maximum speed of the target is $600 \mathrm{~m} / \mathrm{s}$, and the acceleration is $0.1 \mathrm{~m} / \mathrm{s}^{2}$. Figure 7 shows the processing results of different algorithms, among which Figure 7(a) is the results of MTD, from which it can be seen that the accumulated energy of the target signal is smaller and the phenomenon of diffusion occurs; Figure 7(b) is the result of $\mathrm{ACCF}$, from which the target can be observed, but the probability of false alarm is relatively high. Figure 7(c) and Figure 7(d) indicate the processing results of the proposed method, with the speed compensation value to be $300 \mathrm{~m} / \mathrm{s}$. Comparing the results of Figure 7, it can be concluded that the proposed method can detect weak target signals, and that within the allowable range, the larger $\mathrm{P}$ is, the higher the detection probability, which verifies the effectiveness of the proposed method.

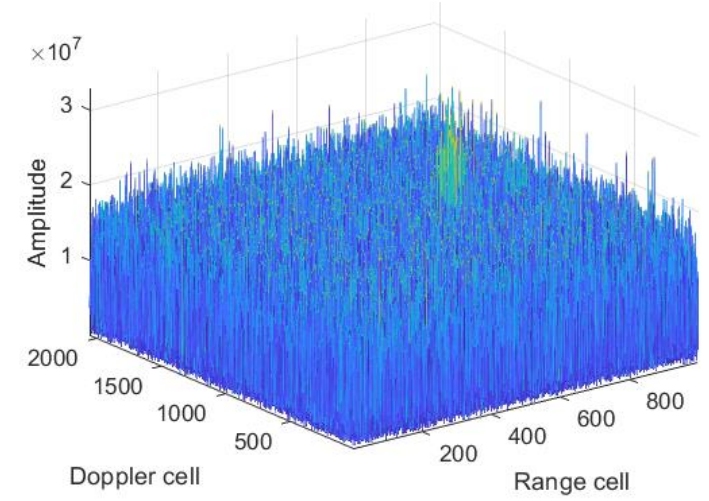

(a)

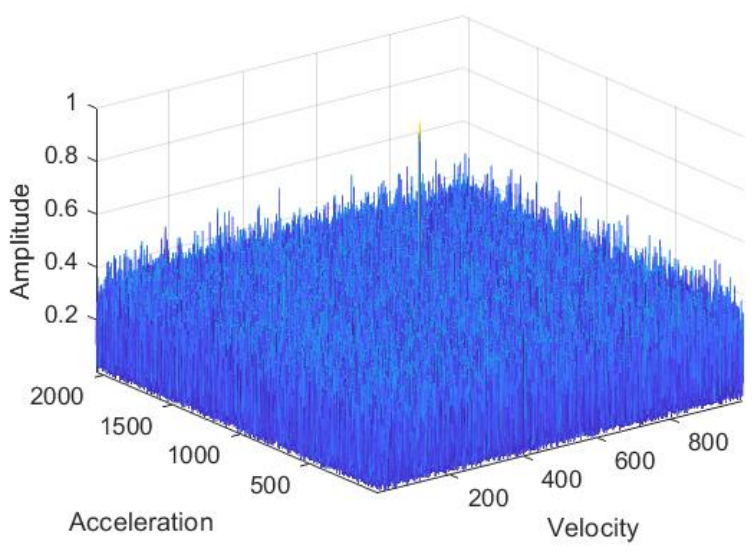

(b)

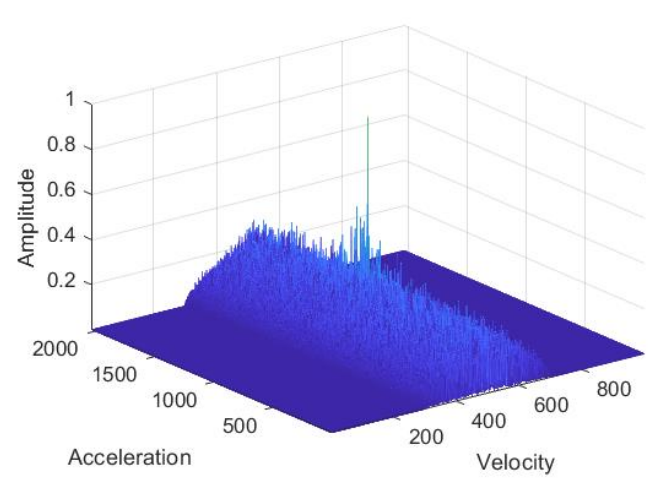

(c)

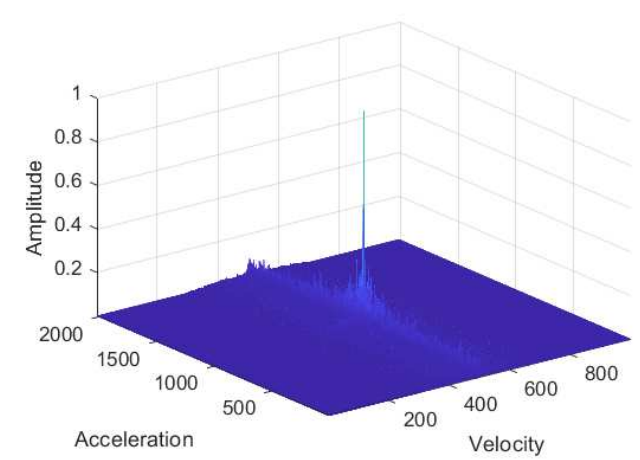

(d)

FIGURE 7. Results of different algorithms for aircraft data. (a) MTD, (b) ACCF, (c) Proposed method( $P=16)$,(d) Proposed method ( $P=32$ ).

\section{CONCLUSION}

Specific to the problems of high computational complexity and poor detection performance for low SNR signals in the existing detection methods for highly maneuvering targets, a coherent accumulation detection algorithm based on SADPT is proposed in this paper, which integrats the advantages of both the parameter compensation method and the DPT method,as follows: 1)It only needs to compensate the target speed and acceleration a few times before using DPT to estimate the target speed and acceleration, and this improves the detection probability and computational efficiency of this type of methods in detecting low SNR signals. 2) The proposed algorithm derives from segmented FFT the preliminary information about the target position, which improves the output SNR; this helps improve the accuracy of parameter estimation and reduce the algorithm , s computational load. 3) Compared with the ACCF algorithm, it improves the detection probability of weak signals. The proposed algorithm is suitable for weak signal coherent accumulation detection over a long time. Acknowledgment

This work was supported by Natural Science Foundation of Shanxi Province of China (No. 202101D121064,201801D 121152). 


\section{REFERENCES}

[1] X. Chen, J. Guan, X. Li, and Y. He,Effective coherent integration method for marine target with micromotion via phase differentiation and radon-Lv's distribution,IET Radar, Sonar Navig. 9(9)(2015)1284-1295.

[2] P. Huang, G. Liao, Z. Yang, X. Xia, J. Ma, and J. Ma,Long-time coherent integration for weak maneuvering target detection and high-order motion parameter estimation based on keystone transform, IEEE Trans. Signal Process.64(15)(2015)4013-4026.

[3] Chen, Xiaolong, Sparse long-time coherent integration-based detection method for radar low-observable manoeuvring target, IET Radar, Sonar \& Navigation .1(14)(2020)538-546.

[4] Sun, Zhi, et al.,Coherent detection method for maneuvering target with complex motions, The Journal of Engineering. 21(10)(2019) 80328036.

[5] J. Zheng, H. Liu, Q. Liu,Parameterized centroid frequency-chirp rate distribution for LFM signal analysis and mechanisms of constant delay introduction,IEEE Trans. Signal Process.65(24)(2017)6435-6447.

[6] X. Li, Z. Sun, W. Yi, Computationally efficient coherent detection and parameter estimation algorithm for maneuvering target,Signal Process.155(2)(2019)130-142.

[7] X. Li, Y. Zhong and B. Hu, Motion Compensation for Highly Squinted SAR data based on Azimuth Nonlinear Chirp Scaling Algorithm, 2019 IEEE 5th International Conference on Computer and Communications (ICCC), (2019)220-224.

[8] Abatzoglou T J, Gheen G O,Range, radial velocity, and acceleration MLE using radar LFM pulse train,IEEE Transactions on Aerospace and Electronic Systems. 34(4)(1998)1070-1083.

[9] R. P. Perry, R. C. DiPietro, and R. L. Fante,Coherent integration with range migration using keystone formatting, in Proc. IEEE Radar Conf., (2007)863-868.

[10] G. Sun, M. Xing, X. Xia, Y. Wu, and Z. Bao, Robust ground moving target imaging using Deramp-keystone processing,IEEE Trans. Geosci. Remote Sens.51(2)(2013) 966-982.

[11] J. Zheng, T. Su, and Q. Liu,ISAR imaging of targets with complex motion based on the keystone time-chirp rate distribution,IEEE Geosci. Remote Sens. Lett. 11(7)(2014)1275-1279.

[12] J. Zheng, T. Su, H. Liu, G. Liao, Z. Liu, and Q. Liu,Radar high-speed target detection based on the frequency-domain deramp-keystone transform, IEEE J. Sel. Topics Appl. Earth Observ. Remote Sens.9(1)(2016) 285-294.

[13] R. Sharif and A. Saman,Efficient wideband signal parameter estimation using a Radon-ambiguity transform slice, IEEE Trans. Aerosp. Electron. Syst.43( 2)(2007) 673-688.

[14] J. Xu, J. Yu, Y. Peng, and X. Xia, Radon-Fourier transform for radar detection, I: Generalized Doppler filter bank,IEEE Trans. Aerosp. Electron. Syst.47(2)(2011) 1186-1200.

[15] J. Yu, J. Xu, Y. Peng, and X. Xia,Radon-Fourier transform for radar detection, III: Optimality and fast implementations,IEEE Trans. Aerosp. Electron. Syst.48(2)(2012) 991-1004.

[16] J. Xu et al.,Radar maneuvering target motion estimation based on generalized Radon-Fourier transform,IEEE Trans. Signal Process. 60(12)(2012) 6190-6201.

[17] J. Zheng, et al,Radar high-speed maneuvering target detection based on three-dimensional scaled transform, IEEE J. Sel. Topics Appl. Earth Observ. Remote Sens. 11(8)(2018) 2821-2833.

[18] X. Li, G. Cui, W. Yi, and L. Kong, Fast coherent integration for maneuvering target with high-order range migration via TRT-SKT-LVD, IEEE Trans. Aerosp. Electron. Syst. 52(6)(2016)2803-2814.

[19] R. Tao, N. Zhang, and Y. Yang, Analysing and compensating the effects of range and Doppler frequency migrations in linear frequency modulation pulse compression radar,IET Radar, Sonar Navig. 5(1)(2011).

[20] X. Rao, H. Tao, J. Su, J. Xie, and X. Zhang, Detection of constant radial acceleration weak target via IAR-FRFT, IEEE Trans. Aerosp. Electron. Syst. 51(4)(2016)214-224.

[21] X. Chen, J. Guan, N. Liu, and Y. He,Maneuvering target detection via Radon-fractional Fourier transform-based long-time coherent integration, IEEE Trans. Signal Process. 62(4)(2014)939-953.

[22] C. Pang, T. Shan, R. Tao, and N. Zhang, Detection of high-speed and accelerated target based on the linear frequency modulation radar,IET
Radar Sonar Navig. 8(1)(2014)37-47.

[23] S. Liu, T. Shan, R. Tao, et al,Sparse discrete fractional Fourier transform ants applications, IEEE Trans. Signal Process. 62(24)(2014) 6582-6595.

[24] C. Pang, S. Liu, Y. Han, High-Speed Target Detection Algorithm Based on Sparse Fourier Transform,IEEE Access. 6(2018)37828-37836.

[25] W. Wu, G. Wang, and J. Sun, Polynomial Radon-polynomial Fourier transform for near space hypersonic maneuvering target detection, IEEE Trans. Aerosp. Electron. Syst. 54(3)(2018)1306-1322.

[26] S. Peleg and B. Friedlander, The discrete polynomial-phase transform,IEEE Trans. Signal Process. 43(8)(1995)1901-1914.

[27] S. Liu, T. Shan, Y. D. Zhang, R. Tao, and Y. Feng, A fast algorithm for multicomponent LFM signal analysis exploiting segmented DPT and SDFrFT,in Proc. IEEE Int. Radar Conf. (2015)1139-1143.

[28] C. S. Pang, S. H. Liu, and Y. Han, Coherent Detection Algorithm for Radar Maneuvering Targets Based on Discrete Polynomial-Phase Transform,IEEE Journal of Selected Topics in Applied Earth Observations and Remote Sensing. 12(9)(2019)3412-3422.

[29] Z. Niu, J. Zheng, T. Su, and J. Zhang, Fast implementation of scaled inverse Fourier transform for high-speed radar target detection,Electron. Lett. 53(16)(2017)1142-1144.

[30] J. Zheng, T. Su, W. Zhu, et al, Radar high-speed target detection based on the scaled inverse Fourier transform, IEEE J. Sel. Topics Appl. Earth Observ. Remote Sens.8(3)(2015)1108-1109.

[31] X. L. Li, G. L. Cui, W. Yi, and L. J. Kong, A fast maneuvering target motion parameters estimation algorithm based on ACCF,IEEE Signal Process. Lett. 22(3)(2015) 270-274.

[32] X. Li, G. Cui, L. Kong, et al, Fast non-searching method for maneuvering target detection and motion parameters estimation,IEEE Trans. Signal Process.64(9)(2016) 2232-2244.

[33] J. Zhang, T. Su, J. Zheng, and X. He, Novel fast coherent detection algorithm for radar maneuvering target with jerk motion,IEEE J. Sel. Topics Appl. Earth Observ. Remote Sens. 10(5)(2017)1108-1119. 